\title{
Hemodynamics and Ventricular Function in the Day-12 Rat Embryo: Basic Characteristics and the Responses to Cardiovascular Drugs
}

\author{
MAKOTO NAKAZAWA, MASAE MORISHIMA, HITOSHI TOMITA, SACHIKO M. TOMITA, AND \\ FUSAE KAJIO
}

Department of Pediatric Cardiology and Division of Cardiovascular Research, The Heart Institute of Japan, Tokyo Women's Medical College, Tokyo, Japan

\begin{abstract}
Fenstic circulation and also looked at the hemodynamic effects of $\alpha$ - and $\beta$-agonists, digitalis, and atrial natriuretic peptide, using a modified organ culture system in which the embryo was placed in oxygenated Hanks' balanced salt solution, blood pressure was measured by a servo-null micropressure system, and blood flow pattern was obtained by a $20-\mathrm{MHz}$ pulsed Doppler velocity meter. The peak pressure was $0.5 \pm 0.04$ (SEM) $\mathrm{mm} \mathrm{Hg}$ at the atrium $(n=6), 2.3 \pm 0.10 \mathrm{~mm} \mathrm{Hg}$ at the ventricle $(n=15), 1.6$ $\pm 0.03 \mathrm{~mm} \mathrm{Hg}$ at the truncus $(n=7)$, and $1.0 \pm 0.05 \mathrm{~mm} \mathrm{Hg}$ at the umbilical artery $(n=21)$. There was a pressure drop from the ventricle to the truncus and then a smaller pressure decrease to the umbilical artery. The atrial a-wave was $20 \%$ of ventricular pressure and ventricular inflow blood flow pattern showed very low early-to-late filling ratio, indicating that the ventricle was stiff. These findings were essentially the same as in the chick embryo. We recorded the ventricular image by using a highspeed video system with a frame rate of $200 / \mathrm{s}$, and the ventricular pressure-area loop showed a triangular shape with short isovolumetric phases, which was different from that of the chick embryo at a similar stage. Isoproterenol increased ventricular pressure
\end{abstract}

Previous studies have shown that basic cardiovascular function becomes established at very early stages of cardiovascular morphogenesis and the embryonic circulation is crucial to ensure normal organogenesis (1-3). The critical role of the embryonic circulation was shown by studies in which altered hemodynamics had adverse effects on the normal growth of the embryo (4-6); thus, stability of embryonic circulation is very important for the normal development and growth of the embryo. Inasmuch as the heart and vessels are not innervated during the early and critically important period of organogen-

Received November 29, 1993; accepted June 15, 1994.

Correspondence: Dr. Makoto Nakazawa, Department of Pediatric Cardiology, The Heart Institute of Japan, Tokyo Women's Medical College, 8-1 Kawada-cho, Shinjukuku, Tokyo 162, Japan.

Supported in part by an Open Research Grant from the Japan Research Promotion Society for Cardiovascular Diseases and Grant-in-Aid for Scientific Research, The Ministry of Education, Science and Culture. from $2.3 \pm 0.1$ to $2.6 \pm 0.1 \mathrm{~mm} \mathrm{Hg}(n=7, p<0.05)$ and decreased umbilical blood pressure from $0.68 \pm 0.05$ to $0.61 \pm$ $0.04 \mathrm{~mm} \mathrm{Hg}(n=7, p<0.05)$, suggesting contraction of the outflow tract, although positive change in pressure per unit time of ventricular pressure did not change. Ventricular negative change in pressure per unit time was increased with isoproterenol from $40 \pm 4$ to $51 \pm 6 \mathrm{~mm} \mathrm{Hg} / \mathrm{s}(n=7, p<0.05)$, implicating improvement of diastolic function. Atrial natriuretic peptide and norepinephrine did not exert any significant responses, which was in contrast to the marked effects of these drugs seen in chick embryos. Acetylstrophanthidin, a rapid-acting digitalis, slowed the heart rate from $184 \pm 4$ to $171 \pm 3 \mathrm{bpm}(n=6, p<0.05)$ but did not change other parameters. In conclusion, basic characteristics of the circulation of the mammalian embryo are similar to those of the avian embryo, but responses to various cardiovascular agents known in the chick embryo cannot be extrapolated to the mammalian embryo. (Pediatr Res 37: 117$123,1995)$
Abbreviations
$\mathrm{Dp} / \mathrm{dt}$, change in pressure per unit time

esis, the circulation should be controlled by other mechanisms that include the Frank-Starling mechanism (7), intrinsic control of heart beats at the optimal rate (8), and possibly circulating amines or peptides $(9-12)$. This information has been obtained from the chick embryo. The chick embryonic circulation, however, is largely different from the circulation of the mammalian embryo because the former is supported by the vitelline vessel system, whereas the circulation of the mammalian embryo is connected to the placenta, which has a sinusoidal structure. Thus, basic characteristics of cardiovascular function and response to various agents may be different in these two species. In addition, defining mammalian cardiovascular condition during early development will soon become a clinical issue because of rapid progress of the technique and knowledge of fetal cardiology and also will be useful for hemodynamic studies in the transgenic mouse. Therefore, we studied the hemodynamic characteristics of the rat embryonic circulation 
and defined the hemodynamic effects of $\alpha$ - and $\beta$-agonists, digitalis, and atrial natriuretic peptide, most of which had marked effects on the circulation of the chick embryo (9-13).

\section{METHODS}

Materials and preparation. Wistar-Imamichi rats were bred overnight, and the day on which pregnancy was confirmed by vaginal smear was considered $\mathrm{d} 0$ of gestation. The details of the method were published elsewhere (14-16). Briefly, on $d$ 12 , pregnant rats were anesthetized with an intraperitoneal injection of sodium pentobarbital $(50 \mathrm{mg} / \mathrm{kg})$. An abdominal incision was made to expose the uterus, and a part of the uterus containing one embryo was taken out and placed in a bath system perfused with oxygenated Hanks' balanced salt solution (Fig. 1). In the bath, a part of the uterus wall was pinned down onto a rubber sheet, and its wall and yolk sac were opened to expose embryos and umbilical vessels.

Basic functional characteristics of the ventricle and circulation. We measured blood pressure at the umbilical artery, truncus, atrium, and primitive ventricle with a servo-null micropressure system (model-900, WPI, Sarasota, FL). After removal of the pericardium, when pressure at the heart itself was measured, a microglass pipette was inserted directly into each chamber or vessel under a dissecting microscope. To compare pressures at different parts, we tried to measure pressure sequentially at two or more sites in one embryo (Table 1 ). We could not obtain data at more than two or three sites in one embryo because puncture of the heart and vessels was technically difficult and took more time than expected or caused bleeding, which deteriorated the physiologic condition of the embryo. Because the umbilical artery pressure was obtained in eight points in this "normal" study, we added 13 points from control data of the pharmacologic experiments (see below). The first derivative of ventricular pressure $(\mathrm{dP} / \mathrm{dt})$ was derived with an electronic differentiator, although its absolute value might not be accurate because the frequency response of our system was not high enough for the rapid heart rate of these

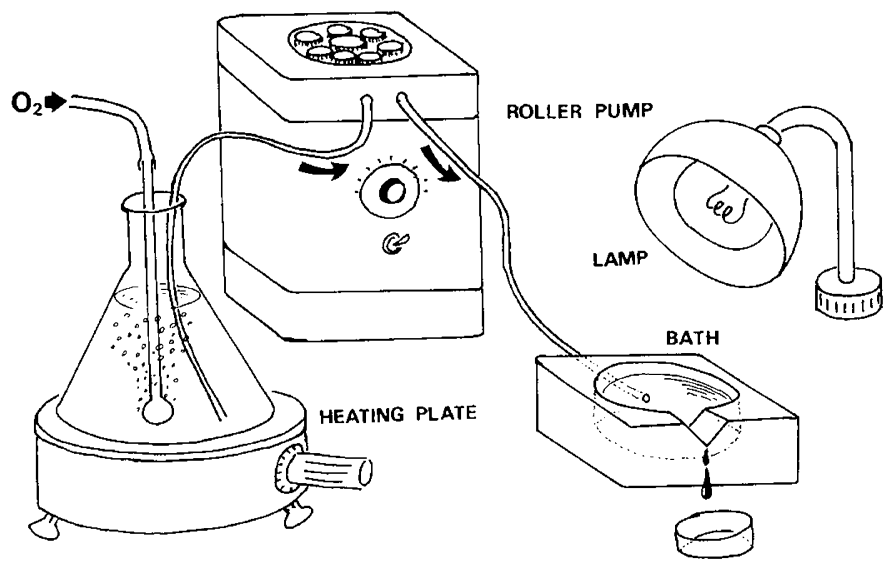

Figure 1. The experimental setup is illustrated. Oxygenated Hanks' balanced salt solution, which was warmed on a heating plate, was pumped into a bath by a roller pump. Temperature of the solution in the bath was finally controlled at $37-38^{\circ} \mathrm{C}$ with a heating lamp. An embryo was kept in the perfusate with the uterus wall pinned down to a rugger sheet at the bottom of the bath.
Table 1. Numbers of embryos for normal hemodynamic data

\begin{tabular}{cc} 
Sites & Numbers \\
\hline Truncus and Ventricle & 7 \\
Truncus + ventricle & 4 \\
Truncus + ventricle + umbilical artery & $3(\mathrm{a})$ \\
Atrium and ventricle & 6 \\
Atrium + ventricle & 3 \\
Atrium + ventricle + umbilical artery & $3(\mathrm{~b})$ \\
Ventricle and umbilical artery & 8 \\
Ventricle + umbilical artery & 2 \\
+ (a) + (b) & 6
\end{tabular}

There were seven embryos from which pressures at both the ventricle and the truncus were sequentially obtained, and in three of the seven, the umbilical artery pressure (umbilical artery) was also taken. (a) and (b) are added to "ventricle and umbilical artery."

embryos. Thus, this value was used only for comparison of different conditions.

In seven embryos, cardiac image was recorded by a highspeed video system (HSV-400, NAC, Tokyo, Japan) with a speed of 200 frames/s. From the images, a ventricular outer (epicardial) border was manually traced and its area was obtained by a computer-assisted planimetry for each frame to construct a time sequence of ventricular area change in a cardiac cycle. Inter- and intraobserver error in this measurement was tested by correlating 20 data points of the area, and correlation coefficients of linear regression were 0.93 and 0.95 , respectively. In these seven embryos, we simultaneously measured ventricular pressure, which was recorded on the video image by using a wave inserter (model V-907, NAC). The pressure measurement system had a time delay of $15 \mathrm{~ms}$ in our laboratory (which was similar to $16 \mathrm{~ms}$ obtained in the system used by Dr. B. Keller; personal communication), and this was taken into account when we constructed pressure-area loop, as was reported by Keller et al. (17) in chick embryos. In the chick embryo, the ventricle is viewed from its right side; thus, the primitive right ventricle is the subject of measurement, whereas the rat embryo is usually in its left-side up position in our experimental setup. Accordingly, the ventricle is viewed from its left side; thus, we measured the area change of the primitive left ventricle. Inasmuch as we did not find notable differences in wall motion between the primitive right and left ventricles, we do not think that the functional characteristics are significantly different. Using a $20-\mathrm{MHz}$ pulsed Doppler velocity meter (University of Iowa, Iowa City, IA), ventricular inflow pattern was recorded, areas underneath early and late (atrial) filling were measured, and then the early filling to the total (early plus late) area ratio was calculated.

Effect of isoproterenol, norepinephrine, atrial natriuretic peptide, and digitalis. To study the effect of cardiovascular agents, the drugs were diluted in $100 \mathrm{~nL}$ of distilled water and injected into the placenta with a microinjector (model 1900, WPI). Doses were $1 \mathrm{ng}$ of isoproterenol, $10 \mathrm{ng}$ of norepinephrine, $1 \mathrm{ng}$ of atrial natriuretic peptide, and $10 \mathrm{ng}$ of acetylstrophanthidin. The doses of isoproterenol, norepinephrine, and atrial natriuretic peptide were similar to those used in chick embryos $(9,11,12)$, whose developmental stages and body weights were almost identical to those of the rat embryos we used $(14,18)$. The dose of digitalis was the minimum dose that 
exerts any significant effect. We did not try a larger dose because even this dosage was already very high and out of physiologic range.

We obtained hemodynamic data while injecting the drugs at an infusion rate of $100 \mathrm{~nL} / \mathrm{min}$. We decided to measure certain parameters for each drug. We obtained ventricular pressure and its $\mathrm{dP} / \mathrm{dt}$ in the study of norepinephrine $(n=8)$ and acetylstrophanthidin $(n=6)$, blood pressure $(n=6)$ and the outflow velocity $(n=8)$ in the study of atrial natriuretic peptide, and all of these measurements in the study of isoproterenol (total $n=$ 20 ). The blood flow velocity at the outflow tract of the primitive ventricle was measured by the $20-\mathrm{MHz}$ pulsed Doppler velocity meter as an index of cardiac output in the study of isoproterenol and atrial natriuretic peptide because these two agents reduced cardiac output in chick embryos (9-11). In this part of study, peak ventricular pressure ranged from 2.3 to 3.1 $\mathrm{mm} \mathrm{Hg}$ in the controls. This variation was the result of each series of experiments being done at different times of $\mathrm{d} 12$; the growth of the embryos is so rapid that the size of the embryos as well as the blood pressure increases within the same day. This range of blood pressure, however, was well below the values at $\mathrm{d} \mathrm{13}$, which ranged from 4 to $5 \mathrm{~mm} \mathrm{Hg}$ (unpublished data, M. Nakazawa).

Data were expressed as mean \pm SEM. Two groups of data were statistically compared by paired or unpaired $t$ test, and $p<0.05$ was considered significant.

\section{RESULTS}

Hemodynamic characteristics of the cardiovascular system. Blood pressure and intracardiac pressure are listed in Table 2. Peak ventricular pressure was higher than truncal pressure, which was higher than the umbilical arterial pressure (Fig. 2). Peak ventricular pressure was constantly higher than peak truncal pressure or umbilical systolic pressure in embryos in which both measurements were done. Atrial a-wave pressure was higher that ventricular end-diastolic pressure $(0.4 \pm 0.04$ $\mathrm{mm} \mathrm{Hg}$ ) in the six embryos from which data were taken at both sites.

The ventricular area increased with the onset of atrial contraction as if the atrial pump actively dilated the ventricle (the ventricular dilatation looked completely passive at this time). After reaching the maximum, the area started to decrease and, after reaching the minimum, it remained small during the early diastolic phase until dilatation with the atrial contraction of the next beat (Fig. 3). Pressure-area loop of the rat embryonic heart was somewhat triangular with short or minimal isovolumetric

Table 2. Blood pressure and intracardiac pressures

\begin{tabular}{lcccc} 
& Atrium & Ventricle & Truncus & $\begin{array}{c}\text { Umbilical } \\
\text { artery }\end{array}$ \\
\hline$n \quad \begin{array}{cccc}\text { Systole } \\
\quad \text { peak) }\end{array}$ & $0.5 \pm 0.04$ & $2.3 \pm 0.10$ & $1.6 \pm 0.03$ & $1.0 \pm 0.05$ \\
$\begin{array}{c}\text { Diastole } \\
\quad \text { (lowest) }\end{array}$ & & $-0.2 \pm 0.02$ & $0.4 \pm 0.03$ & $0.47 \pm 0.03$ \\
End-diastole & & $0.4 \pm 0.02$ & & \\
\hline
\end{tabular}

Pressure mm Hg; mean \pm SEM.

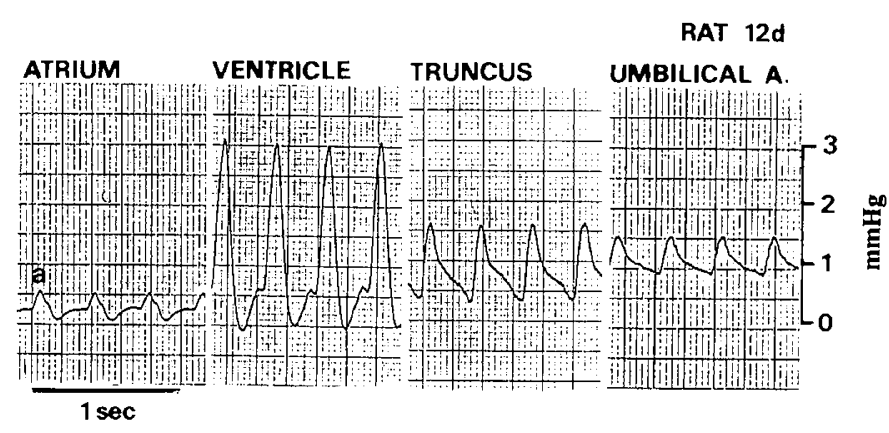

Figure 2. Representative pressure tracings are shown at each part in "normal" condition. Note that there is a pressure drop from the ventricle to the the truncus and then another smaller drop in the umbilical artery. These pressures were taken from different embryos.

systolic and diastolic phases (Fig. 4), which was basically similar to the pressure-volume loop of the mature right ventricle.

To characterize the diastolic property of the primitive ventricle, we calculated diastolic slope of the pressure-volume relation by assuming that volume was equal to area $3 / 2$. The slope was $6.6 \pm 1.6 \mathrm{~mm} \mathrm{Hg} / \mathrm{mL}(n=7)$, which was markedly higher than that $(0.045 \mathrm{~mm} \mathrm{Hg} / \mathrm{mL})$ reported in the mature adult heart (18). Diastolic blood flow pattern at the inflow tract of the primitive ventricle showed that the early filling to the total (early plus late) area ratio was $0.23 \pm 0.03(n=9)$ (Fig. 5).

Effect of isoproterenol, norepinephrine, atrial natriuretic peptide, and digitalis. Isoproterenol produced a reduction of mean umbilical artery blood pressure from $0.68 \pm 0.05$ to 0.61 $\pm 0.04 \mathrm{~mm} \mathrm{Hg}(n=7, p<0.05)$, but the blood flow velocity at the outflow tract of the primitive ventricle did not change significantly $(7.4 \pm 0.6$ to $8.0 \pm 0.7 \mathrm{~mm} / \mathrm{s} ; n=6)$. Heart rate increased from $168 \pm 5$ to $185 \pm 6 \mathrm{bpm}(n=13)$ (Fig. 6). Peak ventricular pressure increased from $2.3 \pm 0.1$ to $2.6 \pm 0.1$ $\mathrm{mm} \mathrm{Hg}(n=7, p<0.05)$, but end-diastolic pressure did not change. Peak positive $\mathrm{dP} / \mathrm{dt}$ of ventricular pressure did not change significantly, whereas peak negative $\mathrm{dP} / \mathrm{dt}$ increased $(p<0.05)$ (Table 3). Norepinephrine did not change ventricular pressure, $\mathrm{dP} / \mathrm{dt}$, or heart rate (Table 3 ). Atrial natriuretic peptide tended to increase blood flow velocity at the outflow tract from $5.4 \pm 0.8$ to $5.8 \pm 0.8 \mathrm{~mm} / \mathrm{s}(n=8,0.05<p<0.1)$ but did not influence the mean umbilical artery blood pressure $(0.60 \pm 0.05$ to $0.60 \pm 0.03 \mathrm{~mm} \mathrm{Hg} ; n=6)$. Acetylstrophanthidin reduced the heart rate by $7 \%$ but did not affect ventricular pressure or $\mathrm{dP} / \mathrm{dt}$ (Table 3 ).

\section{DISCUSSION}

Our study defined the hemodynamic characteristics of the d-12 rat embryo. Mammalian and avian embryos are similar in many aspects; however, they differ in their response to cardiovascular agents, which confirmed our previous finding (16). $\mathrm{Hu}$ and Clark (3) reported that in chick embryos there is a pressure difference between the ventricle and the vitelline artery that is attributable at least in part to the resistance of the aortic arches. Our data also demonstrated that there were pressure differences between the ventricle and truncus, strongly suggesting that the 


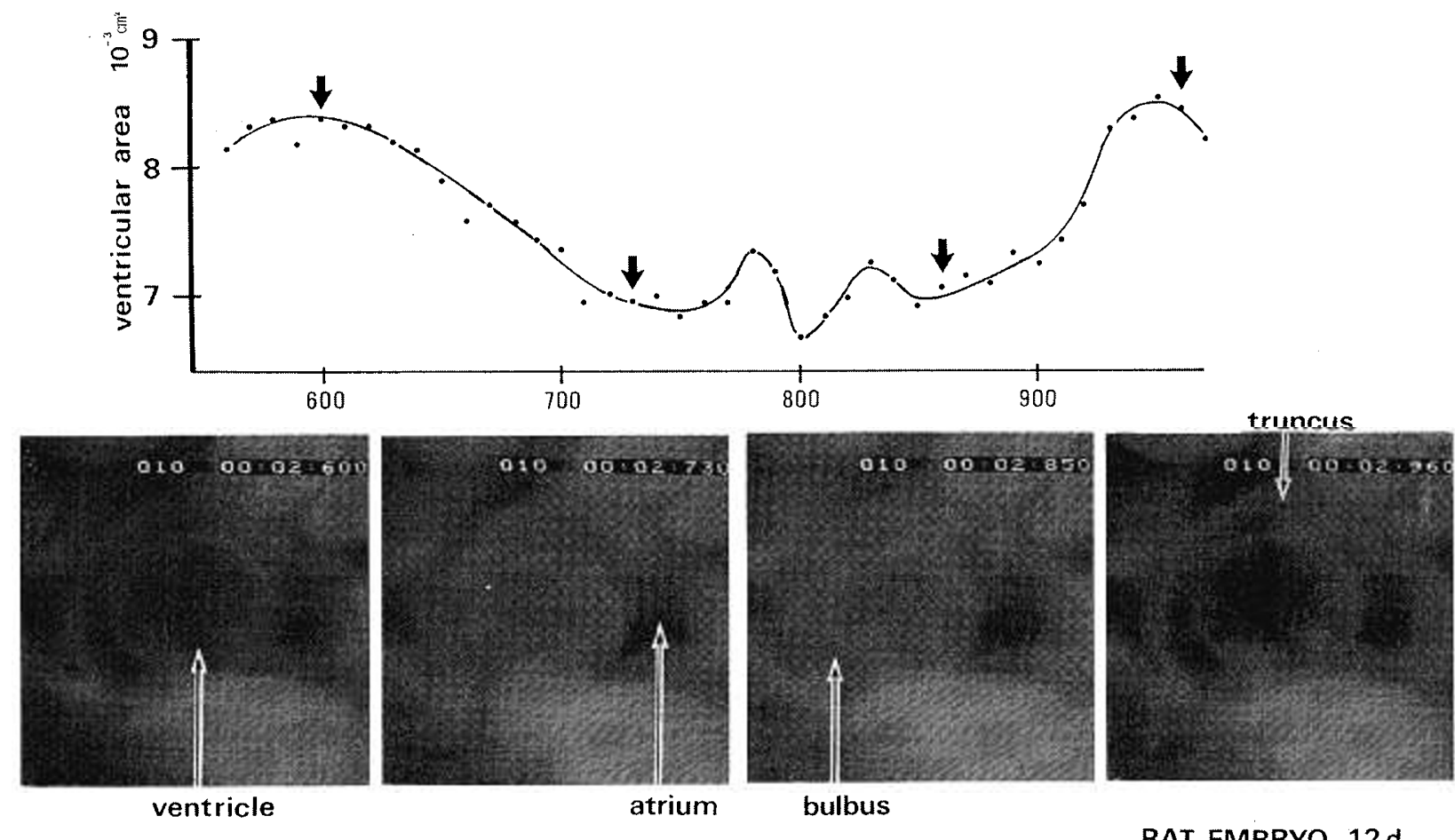

RAT EMBRYO, $12 \mathrm{~d}$

Figure 3. The upper curve shows a time sequence of ventricular area change and the lower four boxes show ventricular images taken from video tape in "normal" condition. In the area change curve, every other point of full data taken in 200 points/s are shown. The points on the curve indicated by arrows correspond to the boxes below each arrow. The numbers on the abscissa of the upper graph indicate frame numbers of video figure shown at right upper corner of each box. Ventricular area increased (first arrow), coinciding with the atrial contraction, and decreased at ventricular contraction (second arrow). It then stayed at a small size and showed fluctuation during diastolic phase (third arrow) until the next atrial contraction that dilated the ventricle (fourth arrow).

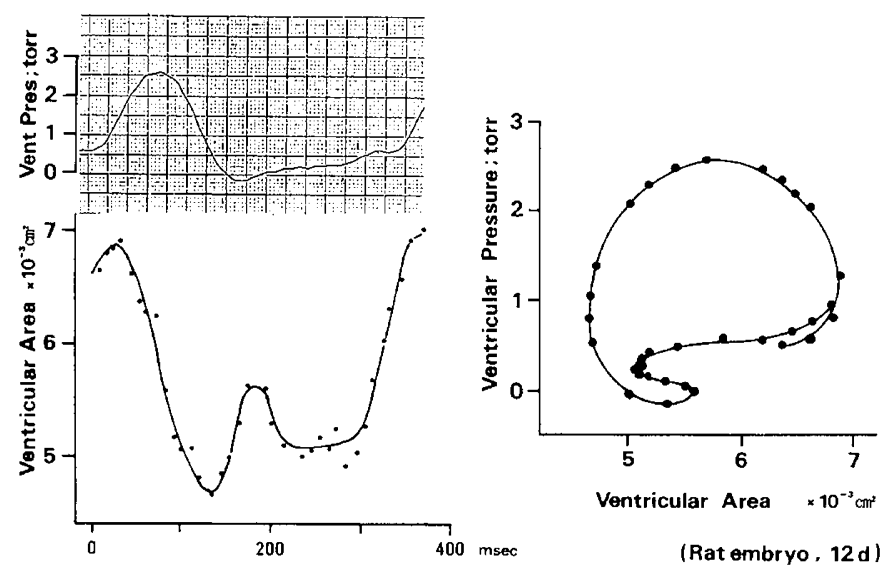

Figure 4. From a simultaneous recording, the ventricular area and pressure loop was drawn in "normal" condition. As is seen in the left-hand figures, as ventricular pressure rises the ventricular area decreases, and there is some fluctuation during diastole while the area itself remains small. In this curve, every other point of full data is shown. The atrial kick is evident in the pressure tracing and corresponds to an increase in the ventricular area from the video film. The pressure-area loop is triangular or globular but not rectangular, and its left-lower corner shows the fluctuation of the area.

pressure drop was brought about by the conal swelling. The conal swelling is not fixed but dynamically changes within a cardiac cycle. At the beginning of ventricular contraction, the outflow tract is still closed by the swelling, thus giving resistance to the flow. This also brings about an isovolumetric contraction phase, although it lasts for only a short period. Then, as the outflow tract opens, the resistance decreases, but

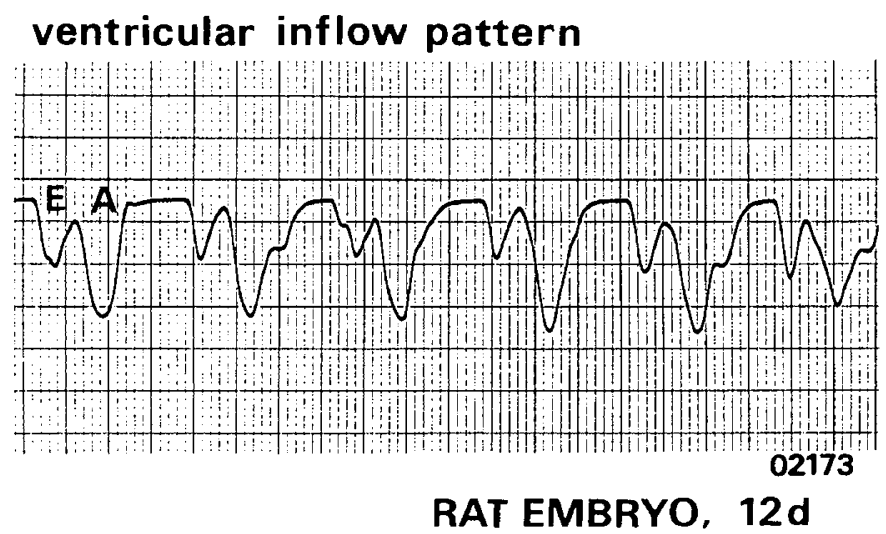

Figure 5. Ventricular inflow pattern, obtained with pulsed Doppler echo in "normal" condition, showed a small early filling $(E)$ with a large late a-wave $(A)$, indicating that the ventricle of this embryo was stiff.

it still remains at a certain level probably because the swelling is not completely compressive, thus producing the pressure drop throughout the ejection period. We wondered whether the pressure drop between the truncus and the umbilical artery is related to physical characteristics of the flow through the very thin vascular system. By using the Poiseuille equation, assuming that this channel is 1 unit of tube, we have found that the pressure difference between the entrance and exit of the tube is in the order of $0.01 \mathrm{~mm} \mathrm{Hg}$. The measured value was 0.6 $\mathrm{mm} \mathrm{Hg}$ on an average; thus, this pressure drop was likely due to the same mechanism as observed in the chick embryo where the aortic arches were considered to produce pressure difference (3). 

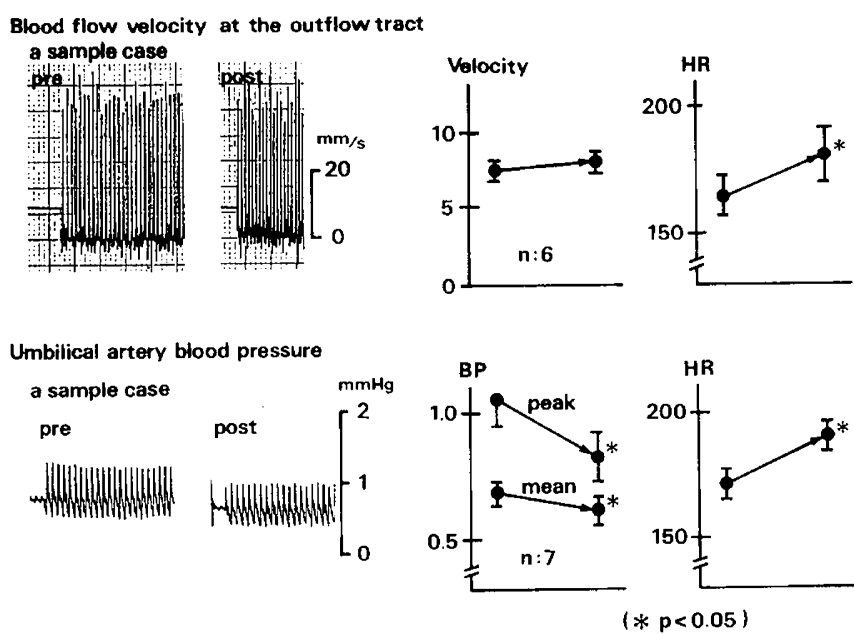

Figure 6. Isoproterenol did not affect the blood flow velocity, a measure of cardiac output, at the outflow tract of the primitive ventricle (top). This drug lowered the umbilical artery blood pressure $(B P$, bottom). Heart rate $(H R)$ increased with isoproterenol. Data are shown as mean \pm SEM.

Table 3. Effects of $\alpha$ - and $\beta$-agonist and acetylstrophanthidin

\begin{tabular}{|c|c|c|c|c|c|}
\hline & \multicolumn{4}{|c|}{ Ventricular pressure } & \multirow{2}{*}{$\begin{array}{l}\text { Heart } \\
\text { rate }\end{array}$} \\
\hline & Systole & EDP & $+\mathrm{dP} / \mathrm{dt}$ & $-\mathrm{dP} / \mathrm{dt}$ & \\
\hline \multicolumn{6}{|c|}{$\begin{array}{l}\text { Norepinephrine } \\
\quad(n=8)\end{array}$} \\
\hline Pre & $3.1 \pm 0.2$ & $0.3 \pm 0.06$ & $39 \pm 4$ & $42 \pm 5$ & $188 \pm 9$ \\
\hline Post & $3.0 \pm 0.2$ & $0.3 \pm 0.06$ & $39 \pm 4$ & $44 \pm 6$ & $196 \pm 7$ \\
\hline \multicolumn{6}{|c|}{$\begin{array}{l}\text { Isoproterenol } \\
\quad(n=7)\end{array}$} \\
\hline Pre & $2.3 \pm 0.1$ & $0.3 \pm 0.04$ & $45 \pm 3$ & $40 \pm 2$ & $172 \pm 8$ \\
\hline Post & $2.6 \pm 0.1^{*}$ & $0.3 \pm 0.03$ & $49 \pm 3$ & $51 \pm 3^{*}$ & $176 \pm 8$ \\
\hline \multicolumn{6}{|c|}{$\begin{array}{l}\text { Acetylstrophantidin } \\
\quad(n=6)\end{array}$} \\
\hline Pre & $2.6 \pm 0.1$ & $0.3 \pm 0.03$ & $45 \pm 3$ & $49 \pm 2$ & $184 \pm 4$ \\
\hline Post & $2.6 \pm 0.1$ & $0.3 \pm 0.03$ & $42 \pm 3$ & $46 \pm 3$ & $171 \pm 3^{*}$ \\
\hline
\end{tabular}

Pressure: $\mathrm{mm} \mathrm{Hg}$; $\mathrm{dP} / \mathrm{dt}: \mathrm{mm} \mathrm{Hg} / \mathrm{s}$; mean $\pm \mathrm{SEM}$. EDP, end diastolic pressure.

${ }^{*} p<0.05$ vs pre.

We also wondered why the normally developing circulation should have the pressure gradient at the outflow tract of the ventricle. Clark et al. (20) demonstrated that constricting the conotruncal region of 21 chick embryos, which resulted in an increase in peak ventricular pressure, caused an increase of ventricular mass compared with sham-operated embryos when examined at later stages (20). On the other hand, reduction of the volume load to the primitive ventricle by continuous administration of verapamil resulted in the growth retardation of the ventricle (21). These studies indicate that an adequate load to the primitive ventricle is required in the normal growth of the heart, and the pressure gradient at the outflow tract seen in our study would be an essential burden to increase the afterload to the ventricle, thus facilitating the normal growth.

The ventricle of the rat embryo was much stiffer than in the mature ventricle. This is also true in the chick embryo $(22,23)$, although the diastolic flow pattern of the rat embryo seemed to be quantitatively different from the data of $\mathrm{Hu}$ et al. (22), who found that the early filling volume was almost identical with the late (active) filling volume in stage 21 chick embryos, whereas our data showed an approximately 1:3 relation. We compared our data with those of stage 21 chick embryos because both are similar in gross morphology such as the degree of looping or the endocardial swelling $(13,17)$. Ventricular area change during the diastolic period is also different in these two species; it increases constantly throughout diastole in the stage 21 chick embryo (16), whereas it remains small until the next atrial contraction in the rat embryo as illustrated in Figures 3 and 4. There was fluctuation in the ventricular area during diastole, which could be related to elastic recoiling or could be only an artifact due to rotation of the heart. Regardless of the reason, it remained small during diastole. This finding is consistent with the difference in inflow velocity pattern. We speculated that this was a species difference. In the d-12 rat embryo, ventricular trabeculation is so heavy that the intraventricular cavity is almost completely obliterated in systole, unlike in the stage 21 chick embryo; thus, we first considered that the difference in trabeculation would explain this finding. However, in the stage 18 chick embryo, the ventricular cavity is completely closed at systole, but the diastolic flow patterns shows that the E-wave is larger than the A-wave (22) and the ventricular area increases throughout diastole (17). Thus, different diastolic characteristics could be due to species difference. Because we were not able to obtain the data in embryos at other gestational days, we do not completely dismiss the possibility that the difference is also due to the different speed in the development of these two species.

The ventricular pressure-area loop was not rectangular and was similar to the shape of the mature right ventricle $(24,25)$. The finding that isovolumetric contraction and relaxation phases were short was somewhat different from that of Keller et al. (17), who found that there were definite isovolumetric phases during a cardiac cycle in the chick embryonic ventricle. In our data, diastolic pressure at the truncus was at almost the same level as ventricular diastolic pressure, indicating that as ventricular pressure rises it exceeds truncal pressure at relatively low pressure, thus ejection starts shortly after the beginning of the systole, which results in the short isovolumetric period. In diastole, as is seen in Figure 4, there is an isovolumetric relaxation phase or a phase with minimal volume change. Immediately after the end of systole, ventricular pressure becomes negative (Fig. 2 and Table 2), which creates a pressure gradient at the atrioventricular cushion and propels blood flow into the ventricle from the atrium. The short isovolumetric contraction phase may be specific in the rat embryo, but we cannot be sure because we did not collect data for other gestational days. This phase may become significant in other developmental stages.

The difference in extraembryonic circulations between the avian and mammalian embryo (the vitelline circulation and placental circulation, respectively) could in part explain the different responses of embryonic hemodynamics to cardiovascular agents as suggested by our previous studies $(15,16)$. It has been reported that in the chick embryo various agents result in significant alterations in blood pressure and cardiac output (9-16), suggesting their role in teratogenecity and in circulatory control before innervation. In chick embryos, isoproterenol and atrial natriuretic peptide reduced cardiac output and 
lowered blood pressure and epinephrine increased blood pressure, all of which were brought about by the drugs' vascular actions (9-12), which were not seen in the rat embryo. We speculate that the lack of apparent changes in blood pressure and cardiac output is due to a large reservoir function of the placental circulation that shadows a subtle change of vascular tone of the embryo itself. These facts indicate that the pharmacologic data of the chick embryo could not be directly extrapolated to the mammalian embryo.

We also tested the effect of cardiotonic agents, because the treatment of heart failure during early days of gestation may become a clinical issue as fetal cardiology advances. In our study, $\mathrm{dP} / \mathrm{dt}$ was used to evaluate ventricular systolic function. This parameter is one of the isovolumetric phase indexes which assumes that the force of the contractile element of the myocardium is directly proportional to the slope of the upstroke of ventricular pressure only during the period when myocardial fiber length is constant. Thus, the use of $\mathrm{dP} / \mathrm{dt}$ should be questioned when there is no isovolumetric contraction phase, and the embryonic rat ventricle had only a short isovolumetric contraction period. In all our subjects, the peak of positive $\mathrm{dP} / \mathrm{dt}$ coincided at ventricular pressure between 20 and $50 \%$ of its peak (data not presented) and, within this pressure range, the change of ventricular area is not large. Thus, it will be acceptable to use the peak $\mathrm{dP} / \mathrm{dt}$ as a parameter of myocardial contractile function, although this application includes an obvious limitation because of the lack of significant length of the isovolumetric contraction phase.

Isoproterenol and digitalis glucoside had neither a positive nor a negative inotropic effect. This was not unexpected because contractile function and responses to inotropic agents are much lower even in late gestation than after birth $(26,27)$, and contractile elements are even more sparse in the embryonic heart (28). It was reported in d-3 chick embryos that isoproterenol increased contractility in the isolated perfused ventricle (29) and that $\beta$-receptor stimulation resulted in a decrease in blood pressure (30), possibly due to dilation of the vitelline vessels $(10-12)$. These studies suggest that $\beta$-receptor is functioning in the cardiovascular system at this stage of development in the chick. Frieswick et al. (29) also reported that isoproterenol increased contractility in the rat embryonic ventricle in a dose- and gestational day-dependent manner (29). The inotropic response was small on $\mathrm{d} 14$ of their study, thus it should be even less or may be negligible on $d 12$, the day we used. Our data that systolic ventricular pressure increased and umbilical blood pressure decreased with isoproterenol (Fig. 6) indicate that the drug has induced a pressure drop somewhere along the blood flow. It could be speculated that isoproterenol modestly increased contractility, but not as much as positive $\mathrm{dP} / \mathrm{dt}$, which caused narrowing of the cylinder-shaped conal region and thus obstruction to the flow. We noted that isoproterenol increased negative $\mathrm{dP} / \mathrm{dt}$. This fact also supported the observation that this $\beta$-agonist had some action on the ventricle, although the mechanism of this action is not clear at present. These discussions should be read with a limited value of application of $\mathrm{dP} / \mathrm{dt}$ as an index of myocardial function as described above.
Digitalis slowed heart rate in embryonic hearts. Paff et al. (13) reported that digoxin given topically slowed heart rate in chick embryos at $36 \mathrm{~h}$ incubation and resulted in heart block in later stages. Although we did not find heart block, negative chronotropism was a common finding. Negative chronotropic effect of digitalis is related not only to its stimulating action of the vagal nerve but also to alternation of ionic flux, thus affecting action potential. Because this stage of embryonic hearts is not innervated (31), the latter mechanism should be responsible for our finding. Although the dose was outside of the pharmacologic range, this mechanism may be of value in the treatment of tachyarrhythmias seen in human fetuses.

Acknowledgments. The authors thank Professor Kazuo Momma for helpful comments and Janet Slater for the language editing of the manuscript.

\section{REFERENCES}

1. Clark ED 1990 Growth, morphogenesis, and function: the dynamics of cardiac development. In: Moller JH, Neal WA (ed) Fetal, Neonatal, and Infant Cardiac Disease. Appleton \& Lange, Norwalk, CT, pp 1-23

2. Clark EB, Hu N 1982 Developmental hemodynamic changes in the chick embryo from stage 18 to 27 . Circ Res 51:810-815

3. Hu N, Clark EB 1989 Hemodynamics of the stage 12 to stage 29 chick embryo. Circ Res 65:1665-1670

4. Rychter Z 1962 Experimental morphology of the aortic arches and the heart loop in chick embryo. Adv Morphogenesis 2:333-371

5. Pexieder $\mathrm{T} 1969$ Blood pressure in the third and fourth aortic arches and morphologic influence of laminar blood stream in the development of the vascular system of the chick embryo. Folia Morphol (Praha) 17:273-290

6. Harh JY, Paul MH, Gallen WJ, Friedberg DZ, Kaplan S 1973 Experimental production of hypoplastic left heart syndrome in the chick embryo. Am J Cardiol 31:51-56

7. Wagman AJ, Hu N, Clark EB 1990 Effect of changes in circulating blood volume on cardiac output and ventricular blood pressure in the stage 18,24 , and 29 chick embryo. Circ Res 67:332-340

8. Cuneo B, Hughes S, Benson DW Jr 1993 Heart rate pertubation in the stage 17:27 chick embryos: effect on stroke volume and aortic flow. Am J Physiol 264:H755H759

9. Nakazawa M, Kajio F, Ikeda K, Takao A 1990 Effect of atrial natriuretic peptide on hemodynamics of the stage 21 chick embryo. Pediatr Res 27:557-560

10. Clark EB, Hu N, Dooley JB 1985 The effect of isoproterenol on cardiovascular function in the stage 24 chick embryo. Teratology 31:41-47

11. Nakazawa M, Miyagawa S, Takao A 1986 Cardiovascular effects of isoproterenol in the stage 21 chick embryo. J Jpn Soc Pediatr Cardiol 2:238-243 (in Japanese)

12. St Petery LB, Van Mierop LHS 1977 Evidence for the presence of adrenergic receptors in 3 day old chick embryo. Am J Physiol 232:H250-H254

13. Paff GE, Boucek RJ, Klopfenstein HS 1969 Experimental heart-block in the chick embryo. Anat Rec 149:217-224

14. Nakazawa M, Miyagawa S, Ohno T, Miura S, Takao A 1988 Developmental hemodynamic changes in rat embryos at 11 to 15 days of gestation: normal data of blood pressure and the effect of caffeine compared to data from chick embryo. Pediatr Res 23:200-205

15. Nakazawa M, Ohno T, Miyagawa S, Takao A 1989 Hemodynamic effects of acetylcholine in the chick embryo and differences from those in the rat embryo. Teratology 39:555-561

16. Nakazawa M, Miyagawa S, Morishima M, Takao A, Ohno T, Ikeda K, Mori K 1990 Hemodynamic effects of cardiovascular agents on the embryonic circulation: a comparative study in chick and rat embryos. In: Clark EB, Takao A (eds) Developmental Cardiology: Morphogenesis and Function. Futura Publishing, Mount Kisco, NY, pp 315-323

17. Keller BB, Hu N, Serrino PJ, Clark EB 1991 Ventricular pressure-area loop characteristics in the stage 16 to 24 chick embryo. Circ Res 68:226-231

18. Sissman NJ 1970 Developmental landmarks in cardiac morphogenesis: comparative chronology. Am J Cardiol 25:141-148

19. Diamond G, Forrester JS, Hargis J, Parmley WW, Danzig R, Swan HJC 1971 The diastolic pressure-volume relationship of the canine left ventricle. Circ Res 29:267275

20. Clark EB, Hu N, Frommelt P, Vandekieft GK, Dummett JL, Tomanek RJ 1989 Effect of increased pressure on ventricular growth in stage 21 chick embryos. Am J Physiol 257:H55-H61

21. Clark EB, Hu N, Turner DR, Litter JE, Hansen J 1991 Effect of chronic verapamil treatment on ventricular function and growth in the chick embryo. Am J Physiol 261:H166-H171

22. Hu N, Connuck DM, Keller BB, Clark EB 1991 Diastolic filling characteristics in the stage 12 to 27 chick embryo ventricle. Pediatr Res 29:334-337

23. Hu N, Keller BB, Taber LA, Clark EB 1990 Diastolic wall modulus and ventricular stiffness in the stage 16 to 27 chick embryo. Circulation 82:III-605 
24. Maughan WL, Shoukas AA, Sagawa K, Weisfeldt ML 1979 Instantaneous pressurevolume relationship of the canine right ventricle. Circ Res 44:309-315

25. Schwiep F, Cassidy SS, Ramanathan M, Johnson Jr RL 1988 Rapid in vivo determination of instantaneous right ventricular pressure and volume in dogs. Am J Physiol 254:H622-H630

26. Nakanishi T, Okuda H, Kamata K, Abe K, Sekiguchi M, Takao A 1987 Development of myocardial contractile system in the fetal rabbit. Pediatr Res 22:201-207

27. Okuda H, Nakanishi T, Nakazawa M, Takao A 1987 Effect of isoproterenol on myocardial mechanical function and cyclic AMP content in the fetal rabbit. J MoI Cell Cardiol 19:151-157
28. Clark EB, Hu N, Dummett JL, Vandekieft GK, Olson C, Tomanek RJ 1986 Ventricular function and morphology in chick embryo from stages 18 to 29 . Am J Physiol 250:H407-H413

29. Frieswick GM, Danielson T, Shideman FE 1979 Adrenergic inotropic responsiveness of embryonic chick and rat hearts. Dev Neurosci 2:276-285

30. Jaffee OC 1972 Effects of propranolol on the chick embryo heart. Teratology 5:153-157

31. Pappano A 1977 Ontogenetic development of autonomic neuroeffector transmission and transmitter reactivity in embryonic and fetal hearts. Pharmacol Rev 29:3-33 